\title{
Kernos
}

Revue internationale et pluridisciplinaire de religion grecque antique

$20 \mid 2007$

Varia

\section{Les fonctions du mythe dans l'organisation spatiale de la cité}

L'exemple de Tanagra en Béotie

Dominique Jaillard

\section{(2) OpenEdition \\ Journals \\ Édition électronique \\ URL : https://journals.openedition.org/kernos/177 \\ DOI : $10.4000 /$ kernos. 177 \\ ISSN : 2034-7871 \\ Éditeur \\ Centre international d'étude de la religion grecque antique}

Édition imprimée

Date de publication : 1 janvier 2007

Pagination : 131-152

ISSN : 0776-3824

Référence électronique

Dominique Jaillard, "Les fonctions du mythe dans l'organisation spatiale de la cité », Kernos [En ligne], 20 | 2007, mis en ligne le 15 mars 2011, consulté le 08 septembre 2022. URL : http://

journals.openedition.org/kernos/177 ; DOI : https://doi.org/10.4000/kernos.177 


\section{Les fonctions du mythe dans l'organisation spatiale de la cité. L'exemple de Tanagra en Béotie}

Résumé : Comment le mythe contribue-t-il à organiser les espaces différenciés d'une cité, le statut de ses eschatia, les rapports entre centres, chora et limites ? L'œuvre de Pausanias, entre témoignage et logique périégétique, propose une certaine pratique du mythe, panhellénique sinon "oikouménique ", qui produit des topographies mémorielles spécifiques. Elle reconstruit l'espace d'une petite cité béotienne, Tanagra, à partir de traditions épichoriques dont elle schématise les structures, laissant transparaitre les manières dont le mythe articule la relation entre la cité, ses dieux et son territoire, dont il définit les qualités des espaces qu'il met en jeu, montagne, littoral, remparts... Entre ce que « disent» les mythes - l'aition d'une fête par exemple - et ce qu'opère la pratique rituelle, des écarts se font jour qui obligent à interroger la part respective du mythe et du rite dans la structuration de l'espace. Cette étude de cas vise à montrer la valeur opératoire de la notion de mythe au sein des processus par lesquels une cité grecque, communauté d'hommes et de dieux liés par des rites, s'approprie symboliquement l'espace sur lequel elle est établie.

Abstract: Myth and the spatial organization of the City: The case of Tanagra. How does Myth participate in the shaping of the different kinds of space constituing a city, whether it be in the definition of its eschatiai or in the determination of the many internal relations that link together its centre, its chora and its limits? I propose here a case study focussed on Pausanias' description of Tanagra in Boiotia, locating the descriptive content of the text within its wider generic logic. Pausanias' panhellenic, if not "oikoumenic", understanding of Myth is active in producing specific memorial topographies. In the case of Tanagra, the text reconstructs the space of the city from a selection of schematized epichoric traditions, outlining the ways in which local myths embody the city's cohesion on the grounds of the diverse categories of space (sea side, mountain, city gates) implied in the narratives. The discrepancies between what the myths are telling and the effective ritual practices call into question the partaking of Myth and Rite in the structuring of civic space.

Loin de dissoudre l'objet mythe, la critique initiée par M. Detienne ${ }^{1}$ oblige l'historien anthropologue de l'Antiquité à affiner un concept dont la valeur opératoire tient à ses facettes multiples, à sa capacité à explorer un champ de

\footnotetext{
${ }^{1}$ L'invention de la mythologie, Paris, 1981. Voir Cl. CaLAmE, « Mythe et rite en Grèce : des catégories indigènes? ", Kernos 4 (1991), p. 179-204; "Le mythe, une catégorie hellène? ", Recherches sur la philosophie et le langage 18 (1996), p. 85-107; «Du muthos des anciens Grecs au mythe des anthropologues », in B. MEZZADRI (éd.), Mythe et mythographie dans l'Antiquité gréco-romaine, Europe 904-905 (2004), p. 8-37. Sur le statut du mythe, voir les remarques récentes de Ph. BORGEAUD, Exercices de mythologie, Genève, 2004, p. 21-31.
} 
pratiques et de savoirs partagés dont les anciens percevaient l'unité relative

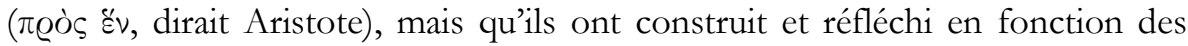
contextes de mise en œuvre, formes de parole ou enjeux à chaque fois spécifiques. Au prix d'un certain flou quant à sa nature - en l'occurrence salutaire le mythe y gagne aussi à ne pas être analysé seulement en termes de récit, mais comme un processus, dont on peut reconnaitre la force opératoire dans un nom ${ }^{2}$, une " concaténation de catégories $»^{3}$, un schème généalogique, un simple lieu, ou dans la relation subtile qui se noue entre des espaces, des objets et des puissances divines ${ }^{4}$. Ce sont certaines de ces dimensions spatiales du mythe que nous souhaiterions approfondir dans le cadre de cette étude ${ }^{5}$. Comment, dans une cité donnée, des mythes fonctionnent-ils comme processus d'investissement et de qualification de l'espace ? Comment contribuent-ils à organiser le territoire de la polis, à en définir, en différencier, les espaces symboliques, à représenter, en relation avec une géographie - mi-imaginaire mi-réelle - et avec les figures du panthéon «local», les caractéristiques et les qualités propres à chaque lieu ? Le mythe concourt à articuler les relations entre une communauté poliade, ses dieux et son territoire, en interférence avec la pratique rituelle, qu'il tend à commenter tout en creusant avec elle des écarts significatifs. Il convient de prendre en compte ce jeu entre mythe et rite, ces zones de non-coïncidence, pour appréhender les pratiques polythéistes dans lesquelles s'insère le processus mythique, et par lesquelles une communauté rassemblant des hommes et des dieux se déploie dans les espaces qu'elle a faits siens. Le choix de Tanagra est en partie le fruit des circonstances. C'est une recherche sur les fonctions d'Hermès dans le panthéon de la petite cité béotienne ${ }^{6}$ qui nous a conduit, pour comprendre le dieu, à explorer la manière dont les mythes y structurent et $\mathrm{y}$ qualifient l'espace. Mais il a aussi d'autres raisons, également d'ordre méthodologique. Comme pour beaucoup de petites cités de Grèce continentale, pour lesquelles la documentation est fragmentaire et éclatée dans le temps, la principale source reste Pausanias ${ }^{7}$, témoin irremplaçable mais œuvre autonome du II ${ }^{\mathrm{e}}$ s. ap. J.-C. ${ }^{8}$. Il faut donc préalablement « décoder » l'écriture périégétique,

\footnotetext{
2 P. Brulé, « Dans le nom, tout n’est-il pas déjà dit ? », Kernos 18 (2005), p. 241-268.

3 J. SCheid et J. Svembro, Le métier de Zeus, Paris, 1994, p. 9-12.

4 Voir, par exemple, l'article toujours pionnier de M. DetiEnNE, «Un éphèbe, un olivier », L'écriture d'Orphée, Paris, 1989, p. 71-84.

${ }^{5}$ Une première version en a été présentée dans le cadre du panel Mythe, dirigé par P. Brulé, à la Celtic Conference in Classics, Rennes, 1-4 septembre 2004.

${ }^{6}$ Cette étude est au cœur de nos recherches sur les Configurations d'Hermès dont le premier volet doit paraittre comme 17e Supplément de Kernos.

7 Pausanias, IX, 20-22.

8 Voir J. Elsner, «Structuring Greece », in S. Alcock, J. Cherry, J. Elsner (éds.), Pausanias. Travel and Memory in Roman Greece, Oxford, 2001, p. 3: "The Periegesis of Greece was written carefully - its every sentence and description deliberately placed according to a conscious pattern of selection that might reveal (if only we could uncover its motivations and underpinnings) the ways the Greek-speakers of the second century A.D. actually thought about their world. »
} 
dégager les traditions et les structures, rituelles, mythiques et panthéoniques, qu'elle laisse entrevoir.

Après la mention d'un sanctuaire extra urbain, le Délion, et quelques considé-

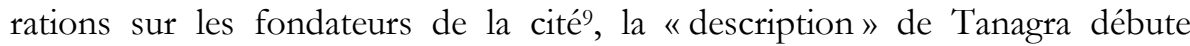
abruptement par la mention elliptique de trois hauts lieux chargés d'échos mythiques : la tombe d'Orion, le mont Kérykion, sur lequel Hermès est né, et le polos d'Atlas où le titan passe pour avoir médité sur le ciel et les profondeurs de la terre ${ }^{10}$. Elle se poursuit avec le temple de Dionysos où sont conservés une statue du dieu, œuvre de Calamis, et un Triton, thauma particulièrement remarquable qui fait l'objet d'une longue digression ${ }^{11}$. L'introduction du temple (naos) de Dionysos marque une inflexion du discours de Pausanias qui, après avoir signalé les principales curiosités de la cité, se concentre sur les sanctuaires urbains. Leur regroupement dans la partie haute fait toute son admiration : les Tanagréens sont les Grecs qui ont le mieux organisé les choses relatives aux dieux en séparant leurs habitations des sanctuaires implantés dans un espace

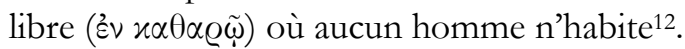

Le logos tanagréen combine deux logiques «descriptives » à la fois antagonistes et complémentaires : l'une, de nature religieuse et mémoriale, dresse l'inventaire des principaux sanctuaires, des «reliques", des mythes et des rites qui leur sont liés, l'autre, de nature topographique, procède en fonction des contiguités ou des proximités. Les temples (naos) de Thémis, d'Aphrodite et de la triade délienne, proches du sanctuaire (bieron) de Dionysos, sont d'abord associés parce que voisins. Les sanctuaires (bieron) d'Hermès Criophoros (à proximité du théâtre et d'un portique), et d'Hermès Promachos, mentionnés juste après ${ }^{13}$, appartiennent également à la partie haute de la cité, réservée aux dieux. Rien, toutefois, ne garantit qu'ils jouxtent les précédents ni qu'ils soient voisins l'un de l'autre. Leur association s'explique plutôt par l'identité du dieu et par les ressemblances entre les mythes qui s'y rapportent. Sans le préciser, Pausanias passe alors aux parties basses de la ville, qui constituent la plus grande partie du site, en contrebas du théâtre. Mais là encore la logique topographique cède le pas au souci antiquaire puisque ne sont mentionnés que les deux monuments les plus à même de marquer l'importance de Tanagra dans la mémoire culturelle commune de l'hellénisme, la tombe de Corinne, qui passe pour y être née, et le

\footnotetext{
${ }^{9}$ Pausanias, IX, 20, 1-2.

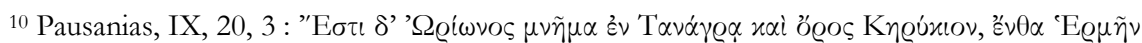

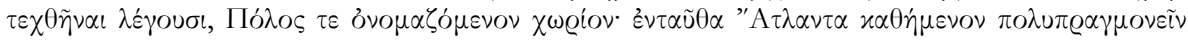

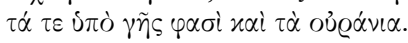

11 Pausanias, IX, 20, 4 - 21, 6. Élien, $H A, 13$, 21, citant Démostratos, un contemporain de Pausanias, qui a visité Tanagra, décrit, avec force considérations, le corps «naturalisé » du triton.

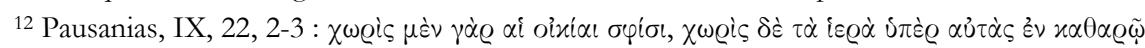

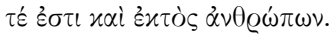

13 Pausanias, IX, 22, 1-2. La plupart des autres sources sont réunies dans D.W. RolLER, Sources and Documents on Tanagra in Boiotia. Tanagran Studies I, Amsterdam, 1989.
} 
gymnase, parce qu'il conserve une peinture figurant la victoire de la poétesse sur le thébain Pindare ${ }^{14}$. Parcours mémorial, paysages divins et fragments de topographie ne cessent d'interférer selon une articulation propre au discours périégétique qui tend dès lors à constituer une construction mythique au second degré.

Pausanias reconstruit un ensemble fragmentaire mais homogène de mythes et de rites qui organisent les espaces de la cité, les qualifient et les différencient les uns par rapport aux autres, selon un processus dans lequel les considérations du périégète - la construction de l'espace mémoriel - se superposent aux données de la tradition mythique locale. Dans la trame du texte, les récits relatifs aux sanctuaires de la ville haute font figure d'aitia : l'épiphanie d'Hermès Promachos prenant la tête des éphèbes explique la présence d'une statue de Calamis dans son sanctuaire, celle d'Hermès Criophoros détournant un loimos, et le rituel éphébique célébré lors de grandes Hermaia civiques. Hermès exerce une action à caractère « répulsif» visant à détourner ou écarter du territoire de Tanagra une menace pressante. Le logos vénérable ${ }^{15}$ ( $\left.\sigma \varepsilon \mu \nu o ́ \tau \varepsilon \varrho o \varsigma\right)$ relatant la victoire de Dionysos sur un triton pieusement conservé dans son temple répond à un schéma comparable. Des dieux se portent aux confins menacés de la cité ou agissent à ses limites. Dionysos et Hermès Promachos interviennent dans les eschatiai, sur le littoral qui fait face à l'Eubée, l'un pour porter secours aux femmes venues se purifier avant la célébration de ses orgia, l'autre pour repousser le débarquement érétrien. Hermès Criophoros écarte le loimos à partir des murailles, à la fois limite majeure qui donne forme à la cité, et lieu obligé de tous les passages.

Si Pausanias sélectionne et schématise ses récits en fonction des memorabilia qu'il met en valeur, il n'en fait pas moins ressortir la cohérence de mythes étroitement articulés à la pratique rituelle, qui explicitent la manière dont les dieux tanagréens assurent la protection du territoire à partir des confins et des remparts, ou articulent les eschatiai et les limites avec le centre cultuel de la cité. Les logoi retenus par Pausanias relèvent en effet d'un ensemble mythique plus large, de tradition proprement tanagréenne, dont l'existence, les structures et le fonctionnement sont corroborés par d'autres sources, en particulier certaines des versions de la fondation de la cité ${ }^{16}$. Mais avant d'analyser la manière dont les « récits-aitia» rassemblés par le périégète structurent une partie des relations entre la communauté tanagréenne, ses dieux et son territoire, il importe de prendre la mesure de la densité mythique du texte de Pausanias, dont la substance et les schémas spatiaux relèvent des traditions mythiques locales sous-jacentes. Les allusions à une version tanagréenne des enfances d'Hermès en constituent

14 Pausanias, IX, 22, 3-4. L'emplacement de la tombe de Corinne est indécidable (¿̉v $\pi \varepsilon \varrho \iota \varphi \alpha v \varepsilon i$ $\tau \tilde{\eta} \varsigma \pi \hat{\lambda} \lambda \varepsilon \omega \varsigma$ ), celui du gymnase est repérable au nord-ouest de la ville près de la porte dite de Délion, voir D.W. ROLLER, "Recent investigations at Grimàdha (Tanagra)», in J.M. FOSSEY (éd.), Boiotia Antiqua, Amsterdam, 1989, p. 156.

15 Pausanias, IX, 20, 4.

16 Plutarque, Moralia, 299c-e. Voir ci-dessous, p. 149 sq. 
l'exemple le plus frappant et, méthodologiquement, le plus instructif. La cité revendiquait la naissance du dieu, à l'instar de sa voisine et rivale Thèbes ${ }^{17}$ et de l'Arcadie qui s'était imposée dans la version panhellénique dont l'Hymne homérique à Hermès est le principal témoin.

Né sur le mont Kérykion, la montagne qui domine Tanagra, le dieu grandit au pied d'un arbousier conservé dans le sanctuaire où il est vénéré sous l'épiclèse de Promachos ${ }^{18}$. En apparence, Pausanias ne dit rien de plus. L'allusion au mythe ne se développe pas en récit. Seuls les lieux sont mentionnés, le mont Kérykion, le sanctuaire du Promachos, et le polos d'Atlas dont la tradition mythique semble unanime à faire le grand-père d'Hermès. Ce sont à la fois des lieux « réels » de la topographie tanagréenne et des lieux «mythiques» tant du point de vue de la construction périégétique que dans la tradition épichorique qui les investit de qualités éminentes et spécifiques. Ces lieux constituent autant de «fragments signifiants » des séquences mythiques qui structurent les différents espaces du territoire de la polis et jouent avec la géographie particulière du site. Il est donc essentiel de prendre en compte les données topographiques dans l'analyse de la perception que les Tanagréens ont pu avoir de l'espace de leur cité, dans ce maillage invisible qui lie les dieux et les lieux, et contribue, en partie du moins, à définir l'identité tanagréenne. La ville est construite à l'extrémité Est du mont Kérykion, sur les premières pentes de la «montagne », selon un type d'implantation assez fréquent en Béotie puisqu'on la retrouve à Orchomène ou à Akraiphnon. Les parties basses sont situées dans la plaine tandis que les parties hautes où sont rassemblés les sanctuaires ${ }^{19}$ forment une première éminence rattachée au reste de la «montagne» par un petit col au relief peu marqué. La cité semble s'intégrer à la chaîne de petites montagnes qui la dominent et en former l'ultime prolongement ${ }^{20}$. Elle est construite au point de rencontre entre plaine et montagne, à l'articulation d'espaces voués à des activités ou à des modes d'exploitation différents, et, au plan mythique, investis

\footnotetext{
17 Pausanias, VIII, 36, 10.

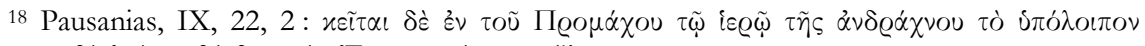

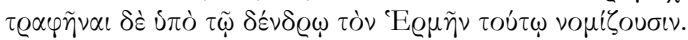

${ }^{19} \mathrm{Du}$ moins depuis la réorganisation du plan de la cité au IVes. Il est toutefois hautement probable que la localisation des sanctuaires d'Hermès et de Dionysos qui nous intéressent ici n'a pas été affectée par la redéfinition de l'espace urbain à l'époque classique. Le peu qui est connu de l'histoire de la cité est désormais aisément accessible (avec bibliographies) dans les courtes synthèses de R. Buck et de J. Fossey, in V. JeAmmet (éd.), Tanagra. Mythe et archéologie, Paris, 2003, p. 22-23 et p. 24-26; pour la topographie, voir les rapports préliminaires que J. Bintliff et son équipe publient régulièrement depuis 2000 dans Pharos et dans Teiresias, ainsi que dans le $B C H$ 2004. Nous remercions le Prof. Bintliff pour son accueil sur le site pendant l'été 2002, et pour ses suggestions stimulantes.

20 Voir J. Bintliff, «The Tanagra Survey Report on the 2000 Season», Pharos 8 (2000), fig. 16, p. 114 (qui propose une modélisation très parlante de la position topographique de Tanagra).
} 
de qualités et de puissances autres ${ }^{21}$. L'arbousier de la courotrophie d'Hermès intégré au sanctuaire du Promachos est situé à l'intérieur de la ville, mais sur les premières pentes qui appartiennent encore à l'espace de l'oros. Ces données topographiques brutes ne suffisent cependant pas à définir les qualités mythiques des espaces en jeu. Rien n'est après tout plus banal que la naissance d'un dieu sur une montagne ou qu'une relique conservée dans un sanctuaire. C'est la confrontation avec les propriétés que les Anciens attribuent à l'arbousier et la comparaison avec les versions parallèles du mythe qui nous a permis de reconstituer la manière dont les récits de l'enfance d'Hermès se tissent dans l'espace tanagréen et contribuent en retour à l'organiser, à le qualifier.

L'andrachnos constitue avec le komaros - l'arbutus des latins - les deux espèces d'arbousier les plus communes en Grèce ${ }^{22}$. Elles se distinguent par des vertus mythiques qui les mettent en rapport avec l'âge d'or et les espaces qui lui sont analogues, confins bienheureux et autres îlots où semblent perdurer les temps primordiaux. Les arbouses sont, avec les glands, si caractéristiques de l'âge d'or que c'est leur commune raréfaction qui y met fin et conduit, dans certaines versions du mythe, Déméter à enseigner l'agriculture aux hommes ${ }^{23}$. Emblématiques de l'automatos bios, des nourritures spontanément et constamment disponibles ${ }^{24}$, elles sont une composante de cette vie amène, d'avant la loi du labeur, dont les poètes de la comédie ancienne se plaisent à brosser le tableau, au bon vieux temps des Titans ${ }^{25}$. À ce titre, elles figurent à côté des glands parmi les nourritures primitives antérieures à l'institution des sacrifices sanglants, lors de la procession du deuxième jour des Thargélies athéniennes ${ }^{26}$. Les jugements

21 Il s'agit avant tout d'un modèle construit par l'imaginaire grec (R. BuXTON, La Grèce de l'imaginaire. Les contextes de la mythologie, tr. fr., Paris, La Découverte, 1996, p. 100-116), les limites exactes entre terres labourées, pâtures et maquis ont pu considérablement varier avec le temps, la prospection conduite par le Prof. Bintliff permettra de se faire une idée plus précise de l'histoire " économique » des pentes de la montagne.

22 Théophraste, Histoire des plantes III, 16; Pline, Histoire naturelle XIII, 120.

23 Virgile, Géorgiques I, 147-149 : cum jam glandes atque arbuta sacrae deficerent silvae; voir Ovide, Métamorphoses I, 101-104; Varron, Économie rurale II, 1; Lucrèce, V, 940-942, qui considèrent l'arbutus comme une nourriture offerte spontanément par la terre, sans qu'il soit nécessaire de la travailler, nourriture des premiers hommes.

24 Phérécrate, Chiron, fr. 158 PCG; Aristophane, Horai (cf. Athénée, 653f), qui mentionne les arbouses parmi les fruits qu'on pouvait trouver à Athènes toute l'année). Pour la place des arbouses dans l'alimentation, A. DALBY, Siren Feasts. A History of Food and Gastronomy in Greece, Londres/New York, 1996, p. 80, note 129.

25 Aristophane, fr. 698 PCG; Cratès, fr. 43 PCG; Théopompe, fr. 68 PCG.

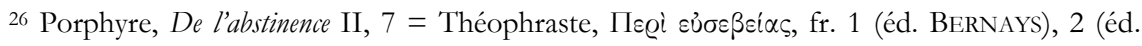
Pötscher). J. Bouffartigue et M. Patillon, CUF, traduisent mimaikula par fraises, ce qui crée une ambiguïté. Le mimaikulon ou memaikulon est selon Théophraste, Histoire des plantes III, 16, 4, le fruit du komaros, ce que confirment un fragment du comique Amphis (fr. 38 PCG), Galien, VI, 620621, Dioscoride, I, 122, Pollux, VII, 144, et Hésychius (s.v. mimakulos) et chez les latins, Pline, Histoire naturelle XV, 98: «les Grecs lui donnent les deux noms de comaros et mimaecylon. » Le rapprochement avec les fraises fragaria se trouve chez Pline (congener), au grand scandale de la 
dont sont l'objet les qualités gustatives et la valeur alimentaire de l'arbouse entre fruit délicieux et denrée à peine consommable - reflètent les ambiguïtés que la tradition attache à l'âge d'or, entre abondance et disette, plénitude et manque ${ }^{27}$. Arbuste de montagne, dans la classification de Théophraste ${ }^{28}$, l'andrachnos est associé aux paysages de maquis et d'agros qui sont le contrepoint des terres labourées. Il participe aussi à la représentation symbolique de la montagne comme une sorte «d'îlot» d'âge d'or où la vie bienheureuse des premiers temps se perpétue sous le règne de Zeus. Des montagnes divines (zatheon), comme le mont Pélion, passent pour fournir spontanément les nourritures les meilleures ${ }^{29}$, et les arbouses de l'Hélicon sont, par leur douceur ${ }^{30}$, comparables aux espèces plantées par l'homme alors que les Grecs tiennent d'ordinaire les fruits cultivés pour meilleurs que les fruits sauvages ${ }^{31}$.

Relique ou bosquet sacré (alsos) 32, l'andrachnos d'Hermès Promachos relève de cette sphère mythique, inscrivant au cœur même de l'espace urbain un fragment de l'espace bienheureux de la montagne qui fait écho à l'âge d'or. Il marque le lien entre la partie haute de la cité, ouverte sur l'espace sauvage, et la montagne divine. Un ensemble de traditions relatives à l'enfance d'Hermès éclaire la configuration mythique que nous venons d'esquisser. Dans la version panhellénique de la naissance du dieu sur le Cyllène, la mère d'Hermès, Maïa, est une nymphe des montagnes ${ }^{33}$, et dans l'Hymne homérique, c'est la grotte natale ellemême qui constitue un "analogue» de l'âge d'or, un espace "ambroisien » comparable aux terres de l'extrême occident que baignent les flots d'Océanos. L'antre de l'Atlantide Maïa sur le mont Cyllène est un double de l'île Ogygie où, selon l'Odyssée, demeure sa sœur Calypso. Antre ou île, ces repaires divins recèlent d'innombrables richesses, les nourritures d'immortalité, nectar et ambroisie, y abondent ${ }^{34}$, mais ils sont comme coupés du reste du monde, isolés

botanique moderne - le fraisier sauvage, le seul connu des anciens, relevant des rosacées, l'arbousier des bruyères - sans doute à cause de leur relative ressemblance (J. ANDRÉ, p. 112).

27 Aristophane, Oiseaux, 240; Théophraste Causes des plantes II, 8, 2; Théocrite, 5, 129; 9, 11; Athénée, 50e-f.; Dioscoride, I, 122 : Galien, peri trophôn 6, 621; Virgile, Géorgiques II, 520; Pline, Histoire naturelle XV, 99; Ano. brevis expos. Virg. Georg. II, 69.

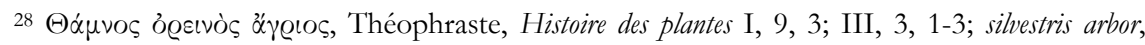
Pline, Histoire naturelle XIII, 120.

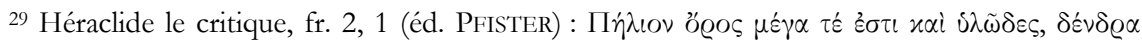

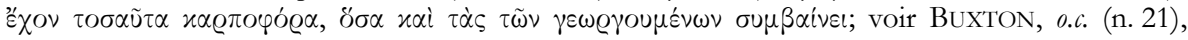
p. 110-113.

30 Pausanias, IX, 28, 1.

31 HP. I, 4, 1; voir M. RocchI, «Le Mont Hélicon, un espace mythique », in A. Hurst, A. SCHACHTER (éds.), La montagne des Muses, Genève, 1996, p. 15-25.

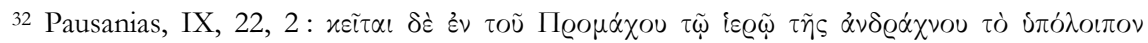

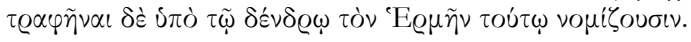

33 Hymne homérique à Hermès, 244; Simonide, fr. 555 (éd. PAGE); scholie à Pindare, Néméennes 2, 17 (éd. DrachmanN, III, 34) et à Lycophron, 219.

34 Hymne homérique à Hermès, 248; Odyssée V, 92-94; 199. 
à la fois des hommes et des dieux ${ }^{35}$. En leurs domaines respectifs, Maïa et Calypso sont exclues du partage des timai qui marque la pleine reconnaissance des prérogatives divines, vers laquelle tendent, à l'inverse, tous les désirs du nouveau-né Hermès. « Nous ne resterons pas ici seuls parmi les immortels, sans prières ni offrandes, alistos et adoretos; en fait de timé, j’aurai la même hosié qu'Apollon ", confesse-t-il à sa mère, après avoir accompli son premier haut fait, le vol des vaches d'Apollon ${ }^{36}$. De même, lorsqu'il se rend chez Calypso, Hermès se plaint qu'aucune cité n'y conduit de belles hécatombes. L'absence de sacrifices est le signe distinctif de cet éloignement, de ce confinement, qui tient ces «cachettes » divines à l'écart de l'espace commun où mortels et immortels entrent en relation ${ }^{37}$. L'antre natal d'Hermès sur le Cyllène, protégé par une bylé profonde ${ }^{38}$, est avant tout le domaine de Maïa, l'équivalent des eschatiai du monde. C'est un espace ambroisien d'un type spécifique, caractéristique du séjour des Atlantides, les filles du Titan Atlas ${ }^{39}$. Or ce dernier est présent à Tanagra, et d'une manière générale les lignées atlantides sont attestées de façon récurrente dans les généalogies de l'Est béotien ${ }^{40}$. La conjonction de la botanique mythique si particulière de l'andrachnos et d'une forte présence atlantide en Tanagride nous incite à reconnaitre dans le Mont Kérykion, à l'onomastique si clairement hermaïque, un espace divin comparable au Cyllène de l'Hymne bomérique.

Divers indices laissent penser que ce paysage mythique "sous influence » hermaïque a pu être lié de manière plus étroite encore avec des mythes mettant en jeu les eschatiai d'extrême-Occident. D'après Servius ${ }^{41}$, le premier arbousier serait né aux extrémités du monde, dans l'île Érythée, du sang du tricéphale Géryon tué par Héraclès. La date tardive de la source interdit toute mise en rapport directe avec Tanagra, mais la présence des os de Géryon à Thèbes, les liens de la cité béotienne avec Ogygos, et l'assimilation de la Cadmée à une île des Bienheureux ${ }^{42}$ montrent que des figures mythiques par ailleurs associées à

\footnotetext{
35 Hymne homérique à Hermès, 5; 142; 172; 228; Od. I, 50; V, 101-102; VII, 246-247.

36 Hymne homérique à Hermès, 167-173.

37 Odyssée V, 101-102.

38 Hymne homérique à Hermès, 228.

39 Atlas fils de l'Océanide Clymène, passe quelquefois, à l'instar d'Océanos, pour le père d'Ambrosia (Hygin, fable 192).

${ }^{40}$ Hyrieus, l'éponyme d'Hyria, une bourgade de la chora tanagréenne, est le fils de Poséidon et de l'atlantide Alcyone; à une vingtaine de kilomètres au nord-est de Tanagra, la cité d'Anthédon, au pied du mont Messapion, sur l'Euripe, doit son nom à un autre fils de Poséidon et d'Alcyone, Anthas (Pausanias, IX, 22, 5).

${ }^{41}$ Servius, Commentaire de l'Énéide VII, 662; voir J. MuRR, Die Pflanzenwelt in der griechischen Mythologie, Innsbruck, 1890, p. 70.

42 Pour Géryon, voir Apostolius, 17, 82, Lucien, Contre un ignorant, 14; pour l'île des Bienheureux, le dossier rassemblé par M. RocCHI, Kadmos e Harmonia. Un matrimonio problematico, Rome, 1989, p. 41-58 et le livre de F. VIAn, Les origines de Thèbes. Cadmos et les Spartes, Paris, 1963, p. 122 124 : Cadmos et Harmonie, après leur exil nord-occidental, sont installés parmi les bienheureux
} 
l'extrême-Occident sont présentes de manière récurrente dans les traditions béotiennes. Érythée est un lieu ambigu qui semble demeurer en deçà du partage du mortel et de l'immortel. Selon Stésichore, Géryon, le maître des lieux, ignore ce qu'il en est de sa propre mortalité, avant qu'il n'en fasse l'épreuve par son combat avec Héraclès ${ }^{43}$. Sa terre natale lui assure, à l'instar du géant Alcyoneus, une immortalité conditionnelle, aussi longtemps qu'une figure extérieure ne fait surgir la possibilité de la mort ${ }^{44}$. Le mythe d'origine de l'andrachnos, arbuste né d'une intrusion brutale de la mort, conduit d'un espace bienheureux, indifférencié, à une claire séparation des conditions mortelle et immortelle. Dans une version parallèle, du sang coule, goutte à goutte, des arbres qui ont poussé sur le tombeau de Géryon, comme s'ils gardaient la marque de la violence et de la mort auxquelles ils doivent leur naissance ${ }^{45}$.

L'arbousier, par son caractère ambigu - entre rupture et mémoire de l'âge d'or -, s'accorde aux mythes de l'enfance d'Hermès et au lieu intermédiaire, entre montagne et cité, où il est conservé. Désormais inséré dans les limites de la ville, il porte les passages entre l'agros montagnard - espace ambroisien où le dieu est né, où la possibilité de la mort est comme suspendue ${ }^{46}$ - et l'espace de la cité, régi par l'économie sacrificielle, et que protège la figure d'Hermès Promachos, adolescente, civique et combattante. Le mouvement qui, dans l'Hymne homérique, mène le jeune dieu du pôle atlantéen de sa mère vers son "destin" olympien de fils de Zeus atteste sa capacité à passer et à faire passer; il marque moins une mutation définitive qu'une capacité à se tenir dans l'entre deux, d'un monde à l'autre. C'est en cette mobilité réversible - selon le temps ou l'espace spécifiquement hermaïque, que peuvent se conjoindre des ordres hétérogènes, s'ouvrir des chemins et des possibles. Lorsque, dans l'Hymne homérique ${ }^{47}$, le

en un lieu qui oscille entre Champs Élysées, extrême-Occident et Thèbes même. Par ailleurs, Aulis, cité voisine de Tanagra - elle lui est rattachée à l'époque de Pausanias -, doit son nom à une fille d'Ogygos (Pausanias, IX, 19, 5), alors que deux autres cités voisines, Hyria et Anthédon, ont pour éponymes des petits enfants d'Atlas. Â Thèbes, Ogygos est un autochtone (Pausanias, IX, 5, 1), et la cité - qui se veut aussi le lieu natal d'Hermès - est traditionnellement qualifiée d'ogygienne. Les Praxidikai, gardiennes des serments, dont Pausanias (IX, 33, 3) a vu le sanctuaire à Haliarte, sont filles d'Ogygos selon Dionysos Chalcidensis, FGH IV 394, 3 (éd. Mulder). Et Ogygie serait un nom ancien de la Béotie (Strabon, IX, 2, 18).

43 Stésichore S 11, 5-26. Voir E. Tsitsibakou-Vasalos, "Stesichorus' Geryoneus S11. 5-26: The dilemna of Geryon ", Hellenika 42 (1991-1992), p. 245-256.

44 Pour Alcyoneus, Apollodore, I, 6, 1. C’est Héraclès encore qui tue le géant en le soulevant du sol auquel son « intuabililité » est liée : C. VIELLE, «L'intuabilité conditionnelle caractéristique de quelques adversaires d'Héraclès. Une approche comparatiste», in C. BONNET, C. JOURDAINAnnequin, V. Pirenne-Delforge (éds), Le Bestiaire d'Héraclès, Liège, 1997 (Kernos, suppl. 7), p. 187-196. Il faut noter que le travail d'Héraclès à Érythée consiste à mener les troupeaux de Géryon vers l'autel d'Héra argienne, où ils seront voués à la mort sacrificielle.

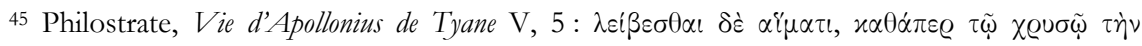

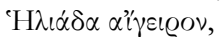

46 Voir notre analyse de l'espace natal d'Hermès, Configurations d'Hermès, cit. (n. 6), chap. I. 1.

${ }^{47}$ V. 112-141. Voir JAILLARD, ibid., chap. II. 1. 
jeune dieu sacrifie nuitamment, seul dans un agros désert, sur les bords de l'Alphée, il mime et réalise les passages qui se jouent autour de la mort sacrificielle, à la fois condition de la vie en cité et ruse du nouveau-né qui anticipe et actualise son intégration dans la communauté olympienne. Il se tient alors, sur le mode qui lui est propre, à la fois à l'intérieur et à l'extérieur du groupe des douze dieux, qui figure le panthéon en sa forme harmonieuse et solidaire ${ }^{48}$. À Tanagra, l'association d'Hermès avec l'andrachnos établit un rapport bipolaire et réciproque, analogue à ceux que l'Hymne met en place, entre montagne bienheureuse et monde de la cité, enfance et adolescence, souvenir de l'Age d'or et intégration à l'ordre politique. Nous approfondirons ce dernier aspect en analysant les épiphanies d'Hermès Promachos et Criophoros.

Pour l'heure, achevons l'exploration des marges. Sur son polos, l'Atlas tanagréen ne fait pas d'abord figure d'ancêtre d'Hermès. Il est le Titan méditant sur « les choses du ciel» et les profondeurs de la terre. Le polos n'est pas un sanctuaire, mais un simple lieu-dit. Aucun culte d'Atlas n'est attesté, ni à Tanagra, ni ailleurs, et le Titan semble rester étranger aux sacrifices et aux honneurs divins. Sa fonction est perçue non comme une timé, mais comme un ponos ${ }^{49}$. Le mythe tanagréen attribue à Atlas des préoccupations (polupragmonein) qui correspondent à la tâche qui lui est assignée aux extrémités de la terre «face au jardin des Hespérides $»^{50}$, supporter la voûte céleste, l'ouranios polos ${ }^{51}$, soutenir de sa tête et de ses bras le vaste ciel, plus précisément: «tenir (echein) les colonnes qui tiennent (echeı) à l'écart (amphis) le ciel et la terre $»^{52}$. Loin d'être un simple porteur du ciel, le Titan est celui qui maintient ouvert l'espace entre ciel et terre, autrefois séparés par la serpe de Cronos. Dans la Théogonie hésiodique ${ }^{53}$, Atlas tient le ciel en s'enfonçant dans les profondeurs de la terre, son exil le projette au fond de l'univers, dans des abîmes terrestres ou marins d'où il dresse la colonne du monde, tandis que sa tête jouxte le ciel ${ }^{54}$. Opérant depuis l'extrême-

48 S. GeOrgOud, «Les douze dieux des Grecs : variations sur un thème », in S. GEOrGOUdI et J.P. Vernant, Mythes grecs au figuré, Paris, 1996, p. 66-70.

${ }^{49}$ Hésiode, Théogonie, 517-520.

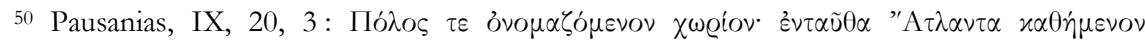

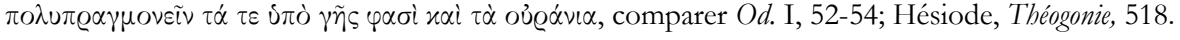

${ }^{51}$ Eschyle, Prométhée, 429, Euripide, fr. 839. 11 (éd. NAUCK ${ }^{2}$ ).

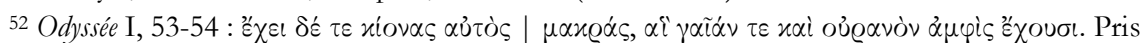
à la lettre les vers de l'Odyssée peuvent être compris de deux façons. Soit les colonnes tiennent à l'écart le ciel et la terre, soit elles les supportent de part et d'autre. Nous pensons avec A. Ballabriga, Le soleil et le tartare. L'image mythique du monde en Grèce archaïque, Paris, 1986, p. 8586, qu'aucune discussion philologique ne peut lever une ambiguité qui explicite la fonction même d'Atlas. Les deux interprétations se complètent, l'une décrit la manière dont Atlas porte à la fois le ciel et la terre, la seconde exprime le caractère ubiquitaire et multidimensionnel du centre atlantéen.

53 V. 736-737.

${ }^{54}$ Eschyle, Prométhée, 349 : Ě $\sigma \tau \eta x \varepsilon$ xiov’ oủ@avoũ. Voir F. Diez de Velasco, « Marge, axe et centre : iconographie d'Héraclès, Atlas et l'axe des Hespérides », Kernos 10 (2000), p. 197-216. 
Occident, à partir des racines de l'univers, Atlas, garant de l'édifice cosmique, tient aussi le ciel en son milieu. Tout comme l'omphalos marin de sa fille Calypso, le polos du Titan constitue un centre excentre ${ }^{55}$.

À Tanagra, le terme polos renvoie à la fois à un emplacement géographique précis et à l'axe du monde que porte Atlas absorbé dans l'accomplissement de sa tâche. Une glose d'Hésychius définissant le polos comme un sommet de forme circulaire $e^{56}$ incite à le localiser dans les parties hautes de la ville ou un peu à l'écart, en direction de la montagne. Dans la topographie tanagréenne, comme dans le mythe, il tend à constituer une manière de centre excentré, de nature purement cosmique, distinct des centres politiques et rituels de la cité. Ce décentrement renvoie, dans l'organisation spatiale du panthéon local, à un préalable cosmique, à une ouverture primordiale de l'espace, qui rend possible la constitution d'un territoire, d'une étendue autonome, structurée et délimitée. Atlas tient les espaces séparés, arrachant le monde à la confusion et à l'indistinction; il autorise la fixation des repères, la différentiation des lieux entre lesquels son petit-fils Hermès passe et fait passer. Dans l'organisation mythique de l'espace tanagréen, le souvenir du Titan semble réitérer ou rappeler les premières mises en ordre du cosmos, en deçà ou aux marges du système sacrificiel et politique de la cité que les cheminements d'Hermès contribuent, au contraire, à mettre en place.

Si la présence d'Atlas à Tanagra renvoie d'abord à ses fonctions cosmiques, une attention à l'onomastique géographique et aux menus indices que livrent des fragments de "description» du territoire tanagréen incitent à formuler l'hypothèse d'une présence titanique assurant une forme de continuité avec l'âge d'or. Le nom de la rivière Asopos, qui joue un rôle fondamental dans les généalogies tanagréennes ${ }^{57}$, se retrouve à Sicyone, la Mékoné hésiodique, lieu étroitement associé au frère d'Atlas, Prométhée. Les deux fils de Japet, exilés aux deux extrémités de l'univers, sont présents sur les rives des deux Asopos dans des configurations mythiques parallèles ${ }^{58}$. À Sicyone, Prométhée enseigne aux hommes la science des levers et des couchers des astres, signes sûrs du passage des saisons ${ }^{59}$, tandis qu'à Tanagra, Atlas s'absorbe dans ses méditations cosmologiques. Des traditions hellénistiques en font un astronome ${ }^{60}$ et un

55 BALlabriga, o.c. (n. 52), p. 75-102.

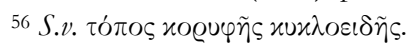

57 Corinne, fr. 654.

58 L'existence d'une koiné mythique entre Béotie orientale et Sicyone est confirmée par des parallèles frappants entre les généalogies des deux régions. Voir VIAN, o.c. (n. 42), p. 194-198 et B. SERGENT, «Les petits nodules et la grande Béotie. III », REA 101 (1999), p. 309-329.

59 Eschyle, Prométhée, 454-458. À Titané, près de Sicyone, un autochtone du nom de Titan, passe pour avoir résidé sur un promontoire élevé d'où il observait le ciel et les saisons (Pausanias, II, 11, 5).

60 Xénagoras, FGrH 31 F 13 (Scholie à Apollonius de Rhodes, IV, 264); Diodore de Sicile, III, 60, 2 et IV, 27, 4-5; Servius Commentaire de l'Énéide I, 741. 
observateur des astres dans sa lointaine et solitaire Hespérie ${ }^{61}$. Mais la relation des deux Asopie à la perpétuation d'un âge d'or titanique se joue d'abord autour des propriétés attribuées aux deux chora, l'une et l'autre associées à une blancheur chargée de référents mythiques. À la terre blanche de l'Asopia sicyonienne dont la fertilité proverbiale rendait la culture aisée $e^{62}$ répond la blanche apparition de Tanagra dans la description du périégète hellénistique Héraclide le

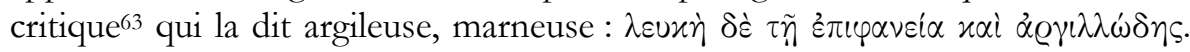
Les deux terres sont à la fois des lieux de rupture et de continuité avec l'âge d'or, comme l'attestent, à Tanagra l'ambiguité de l'andrachnos et, à Mekoné, le mythe de Prométhée. Or, comme l'a montré P. Ellinger ${ }^{64}$, la terre blanche de Sicyone, liée aux Titans, n'a pas complètement rompu avec l'âge d'or, et le paysan y échappe, par la richesse du terroir, au ponos et aux misères de l'âge de fer. Une même impression de richesse aisée, de simplicité et d'harmonie, se dégage de la description de Tanagra chez Héraclide ${ }^{65}$ :

La route passe à travers des oliveraies et des terres boisées, totalement libres de la peur des voleurs. (...) Il n'y a pas une grande abondance de céréales, mais le vin est le meilleur de Béotie. Les habitants brillent par leurs biens et sont simples dans leur vie, tous sont paysans et non artisans. Ils observent scrupuleusement la justice, la parole donnée, l'hospitalité. À ceux de leurs concitoyens qui sont dans le besoin et aux étrangers qui arrivent chez eux, ils offrent ce qu'ils ont et partagent librement. L'injustice et la cupidité leur sont étrangères, et de toute la Béotie, c'est pour les étrangers la cité la plus sûre où séjourner. C'est qu'à cause de l'indépendance et de l'amour du travail des habitants, il y a chez eux une franche haine du mal.

Les deux terres ont conservé des qualités - fécondité du sol, vertus des habitants, simplicité de mœurs... - caractéristiques du règne de Cronos ou des terres titaniques associées à des autochtones primordiaux, ces êtres de chaux qui vivent en deçà d'un partage rigoureux des conditions mortelle et immortelle. Mais sur les bords des fleuves Asopos, cette « continuation » de l'âge d'or se définit dans les termes mêmes de la Diké hésiodique, conformément aux partages institués par le règne de Zeus et la Déméter du travail agricole. Une construction mythique complexe liant figures titaniques, terre blanche et persistance de l'âge d'or rend raison des singularités des descriptions périégétiques et des positions attribuées aux fils de Japet. La présence titanique garantit une

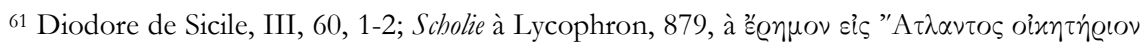
nous remercions B. Sergent qui nous a signalé ce texte.

62 Zenobius, III, 57 (éd. Leutsch-SCHNEIDEwin, I, p. 70).

${ }^{63}$ Fr. 1, 8-10 (éd. Pfister) : cf. B. MALLET, Le journal de voyage d'Herakleides, thèse, Lyon 1982.

${ }^{64}$ P. Ellinger, La légende nationale phocidienne, Athènes/Paris, 1993 (BCH, suppl. 27), $2^{\mathrm{e}}$ partie, chap. 3, " Naître de la chaux : Le pays de la terre blanche », p. 95-104.

${ }^{65}$ Reste entre les descriptions des deux Asopia une différence d'accent. Les sources insistent plutôt sur la surabondance des biens que produit la terre sicyonienne et sur la simplicité de la vie tanagréenne. Qu'une description d'époque hellénistique mette si peu en valeur l'artisanat tanagréen semble confirmer la prégnance des schémas mythiques dans la construction d'Héraclide!
} 
forme de prospérité liée à la fois à la fertilité de la terre et à un savoir astronomique. Cette double action définit aussi une polarité spatiale, entre un point haut d'où le Titan observe le ciel, éventuellement en relation avec Hélios ${ }^{66}$, et la chora «blanche », fertile et cultivée. L'Atlas tanagréen n'est pas seulement le pôle cosmique excentré auquel répond le centre cultuel et politique de la cité, il porte la mémoire féconde de la succession des temps et des règnes divins, comme une strate plus « archaïque » sur laquelle se construit la prospérité de la communauté.

Cette première analyse des espaces mythiques tanagréens, depuis le mont Kérykion et le polos d'Atlas, dessine un paysage investi de puissances dont le jeu détermine en profondeur les manières dont fonctionne l'espace. À considérer le réseau complexe des mythes qui en définissent les qualités, les eschatiai cessent d'apparaitre comme une catégorie homogène; ils revêtent des visages multiples, différenciés; ils ne se laissent pas circonscrire exclusivement en termes de confins géographiques du territoire ou d'opposition du sauvage et du cultivé ${ }^{67}$. La même montagne porte le centre cosmique dont Atlas est en charge, «l'ailleurs » ambrosien des Atlantides, ou l'espace de l'adolescence qui est aussi celui des confins maritimes où les éphèbes combattent sous la conduite d'Hermès. Ces rivages de l'Eubée, où paissent les troupeaux ${ }^{68}$, sont aussi le lieu de surgissement du monstre marin, adversaire de Dionysos... À travers l'arbousier du Promachos, les eschatiai investissent le cœur de la cité, elle-même implantée dans un espace transitionnel, entre chora cultivée et montagne divine. Mais conservé dans le sanctuaire d'Hermès, divinité médiatrice par excellence, l'andrachnos « présentifie » la possibilité d'articuler ce qui est disjoint, hétérogène, cette capacité hermaïque de se tenir à la fois au centre et aux marges, avant et après, dedans et dehors.

Ces relations entre des eschatiai aux figures multiples et le centre urbain, délimité par ses murailles et ses portes, et son clair partage entre espace des hommes et espace des dieux, structurent les mythes étiologiques mentionnés par Pausanias. Nous pouvons désormais les analyser en marquant leur place dans le tissage mythique de l'espace tanagréen, au sein duquel le sanctuaire d'Hermès Promachos joue un rôle privilégié, en liant l'enfance "montagnarde » du dieu et ses combats éphébiques aux franges maritimes du territoire. Le récit

66 Voir Ellinger, o.c. (n. 64), p. 95, avec le parallèle de Titané.

67 Sur les usages du terme eschatia, notamment dans les sources épigraphiques, voir I. POLINSKAYA, "Liminality as Metaphor. Initiation and the frontiers of Ancient Athens », in D.B. DODD et C. FARAONE (éds.), Initiation in Ancient Greek Rituals and Narratives. New Critical Perspectives, Londres/New York, 2003, p. 96-98: «the epigraphic evidence points to the relativity of a spatial term such as eschatia, as we confront the picture of Attic lands with numerous eschatiai scattered throughout, and not only along Attica's external borders - a use that reflects a multiplicity of spatial perspectives ", qu'il nous semble important de réarticuler à leurs dimensions imaginaires et mythiques.

68 Pausanias, IX, 20, 4. 
du périégète ne manque pas de surprendre. Un Hermès combattant est chose rare, l'arme qu'il brandit plus surprenante encore, un strigile, instrument d'ordinaire associé à la cité en paix ${ }^{69}$, à la vie du gymnase ${ }^{70}$, aux activités agonistiques et à l'éducation des éphèbes. Comme pour l'andrachnos, c'est en analysant l'ensemble des valeurs dont l'objet est investi que nous pourrons éclairer l'usage inhabituel qu'Hermès en fait. Le strigile, emblème éphébique par excellence ${ }^{71}$, est étroitement lié à l'acquisition des vertus qui conviennent à de futurs hoplites et citoyens, à un certain modèle de sagesse civique. Une anecdote de Philostrate qui met en scène un gymnaste suivant à Olympie, strigile en main, la course de l'athlète qu'il a entraîné, nous semble particulièrement significative. "Certains racontent qu'à Olympie le gymnaste tua l'athlète avec un strigile aiguisé parce qu'il n'avait pas fait montre d'assez d'endurance pour obtenir la victoire. Contre les mauvais athlètes, que le strigile soit une épée, qu’à Olympie le gymnaste ait quelque pouvoir à côté du juge ! $»^{72}$.

La fermeté, la patience et la persévérance qui ont fait défaut à l'athlète de Philostrate, au lieu où elles auraient dû briller de tout leur éclat, définissent la philoponia, une des vertus éphébiques qu'Hermès juge lors des athla organisés à l'occasion des Hermaia du gymnase et des concours de beauté qui leur sont associés $^{73}$. Hermès, le cosmète par excellence ${ }^{74}$, veille de son regard scrutateur

\footnotetext{
${ }^{69}$ Aristophane, Cavaliers, 579-580 : «si jamais la paix revient et met un terme à nos peines, ne nous enviez point nos longs cheveux et nos membres frottés au strigile. »

${ }^{70}$ Platon, Charmide, 161e; Hippias mineur, 368c et scholie; Aristophane, fr. 214 PCG; Diphilos, fr. 51 PCG; Timée, 566 F 26c (éd. JACOBY). Voir E. KOTERA-FEYER, Die Strigilis, Francfort, 1993 (Europaïsche Hochschulschriften Archälogie, 38, 43).

71 À propos des strigiles figurés sur les stèles funéraires représentant de jeunes hommes et leur fonction dans les tombes, voir KOTERA-FEYER, o.c. (n. 70), p. 75-139; pour Tanagra, A. DE RIDDER, Catalogue des bonzes de la société archéologique d'Athènes, Rome, 1894 (BEFAR, 69), nº 534, 541 (représentation d'un éphèbe en marche tenant un strigile dans la main droite), 570.

72 Philostrate, Gymnastique, 18.

73 Voir notamment Ph. Gauthier, M.B. Hatzopoulos, La Loi gymnasiarque de Béroia, Athènes, 1993 (Meletemata, 16), document particulièrement suggestif pour reconstituer l'organisation des Hermaia. Le jugement du concours éphébique est présidé par Hermès, à qui est destiné le sacrifice initial; les juges des épreuves prêtent serment par Hermès. Le prix de philoponia est décerné à « celui qui paraît s'être entraîné au cours de la présente année en se donnant le plus de peine ». Voir aussi L. ROBERT, Hellenica XI-XII (1960), p. 342-49. Un décret d'Éphèse accorde la

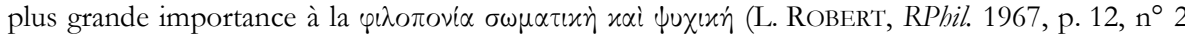
[Opera Minora Selecta V, p. 352]). L'exercice au gymnase peut être désigné par la simple expression Hermeiai melesthai, KAIBEL 247, 5. Dans la loi de Béroia, les vertus que sanctionnent les différentes épreuves constituent la beauté de l'éphèbe (voir N.B. CrowtHER, "Male Beauty Contests in Greece: The Euandria and Euexia », AC 54 [1985], p. 285-291 et «Euexia, Eutaxia, Philoponia: three Contests of the Greek Gymnasium », ZPE 85 [1991], p. 301-304).

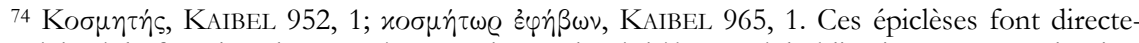
ment écho à la fonction des cosmètes en charge des éphèbes, et à l'obligation pour ces derniers

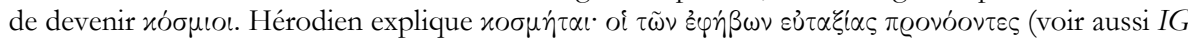
$\mathrm{II}^{2}, 1008$, 55). Sur la présence probable d'un cosmète à Tanagra, voir L. ROBERT, RPhil 1939, p. $122-128$
} 
et vigilant d'episkopos gumnasion 75 à ce que les éphèbes acquièrent les vertus

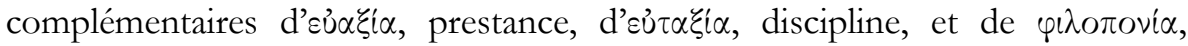
endurance, qui les prépareront à la discipline hoplitique et à la vie kata kosmon des hommes d'âge mûr. À Tanagra, le plus bel éphèbe célèbre les rites en l'honneur d'Hermès, Criophoros cette fois, à l'occasion des grandes Hermaia civiques ${ }^{76}$.

L'Hermès Promachos de Tanagra a toutes les vertus du meilleur éphèbe, hégémon conduisant ses compagnons, repoussant l'ennemi dans un élan agonistique, en un coup de main rapide ${ }^{77}$. La brusque apparition des jeunes Tanagréens qui se portent à la rencontre de l'ennemi produit un effet de surprise qui

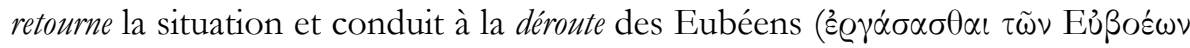
$\tau \varrho \circ \pi \eta \dot{\nu} \nu$, en une trope typiquement hermaïque. Dolos du dieu, le pacifique strigile se «retourne» dans l'émulation du combat en arme redoutable, de même que l'éphèbe qui fait l'épreuve de ses qualités agonistiques se transforme jusqu'à se qualifier pour assumer une place de choix dans la cité. Signe à la fois de la position encore inférieure de l'éphèbe ${ }^{78}$ et des vertus qu'il révèle dans l'agon, le strigile peut apparaître comme une arme adéquate au futur citoyen et au destin qui lui est promis, et l'espace liminal des confins comme un lieu propice à ce retournement ${ }^{79}$.

Dans une représentation du territoire que construisent conjointement l'expérience, la mémoire historique et le mythe, les Tanagréens perçoivent le littoral face à l'Eubée comme un point sensible, le lieu d'une invasion toujours possible par un ennemi emblématique, Érétrie. La place de la cité eubéenne dans l'image que les Tanagréens se font des menaces qui pèsent sur leur territoire est confirmée par un bref aition des scholies à Lycophron ${ }^{80}$. "Parmi les Béotiens, Hermès Leukos, Blanc, est honoré (timatai), car lorsque les Tanagréens subirent l'attaque des Érétriens, ils égorgèrent (esphagiasan) un garçon et une

75 Anthologie Palatine XI, 176, 1; POxy. VII, 1015.

76 Pausanias, IX, 22, 1.

77 Pour comparaison, voir, par exemple, le récit d’Éphore, 105 F 2 (éd. JACOBY) : «Quand Orthagoras sortit de l'âge des enfants et qu'il devint un des peripoloi qui gardaient le pays, une guerre ayant opposé les Sicyoniens aux Pelléniens, il était dans toutes les circonstances actif et bienveillant. Quand les Pelléniens firent incursion et attaquèrent à l'improviste, il porta assistance, tua quelques ennemis et se distingua parmi tous les peripoloi. En récompense de ses prouesses, les Sicyoniens le nommèrent péripolarque; aussitôt après cette distinction, il vainquit les ennemis d'une manière encore plus brillante, de sorte qu'il se concilia l'estime de beaucoup de citoyens et les fit entrer dans son parti. »

78 Voir Artémidore, Onirocritique I, 64. Dans le récit de Philostrate, c'est le maitre qui tient le strigile, comme un instrument d'éducation et de sanction.

${ }^{79}$ Ailleurs, c'est le déguisement des éphèbes en femmes, qui constitue la ruse et prépare le retournement de la situation. Solon, Plutarque, Solon, 8, passe pour avoir envoyé de jeunes Athéniens encore imberbes près du cap Colias, à Halimous, afin d'y porter secours aux Athéniennes qui célébraient les Thesmophories. Les éphèbes déguisés forment un chœur et repoussent les Mégariens débarqués de la mer.

80 Scholie à Lycophron, 679. 
jeune fille conformément à un oracle, et en conséquence, instituèrent le culte d'Hermès Leukos». Différence significative toutefois, le scénario d'une aparché prise sur la jeunesse de la cité ne renvoie pas à une guerre agonistique entre jeunes mais à un conflit où il y va de la survie même de la communauté et de sa capacité à demeurer sur la terre où elle est implantée. Une complémentarité peut toutefois s'instaurer entre le sacrifice qui pérennise l'appropriation du territoire et sa défense par les éphèbes. À Athènes, les éphèbes prêtent serment au lieu même où Aglauraos s'est sacrifiée pour le salut d'Athènes, au pied de l'acropole, lors de la guerre entre Érechthée et Eumolpe ${ }^{81}$.

Les limites maritimes de la Tanagride, où l'on peut situer la rencontre du Promachos et des Érétriens, sont une de ces zones frontières, favorables aux contestations mais aussi aux contacts ainsi que l'atteste la présence du sanctuaire extra-urbain du Délion, fréquenté à la fois par des Béotiens et des Eubéens $^{82}$. Des jeux s'y tiennent qui contribuent à en affirmer la vocation régionale. Une inscription inédite ${ }^{83}$ montre qu'au $\mathrm{II}^{\mathrm{e}}$ siècle av. J.-C., ils étaient conjointement organisés par les Tanagréens et les autres cités béotiennes ${ }^{84}$. Mais, pour notre propos, l'apport le plus important du nouveau document tient aux relations étroites, sans doute rituelles, qui se nouaient entre le sanctuaire du littoral et la cité à l'occasion de l'agon. Une trapéza, vraisemblablement la table requise pour l'accomplissement de certains des sacrifices pratiqués au Délion, était transportée depuis Tanagra puis rapportée en ville après usage ${ }^{85}$. On peut faire l'hypothèse qu'il s'agit d'une table empruntée au sanctuaire urbain de la triade délienne que Pausanias mentionne dans la partie haute de la ville ${ }^{86}$. À la différence de l'autel, la trapeza apparait comme l'élément mobile du rituel ${ }^{87}$. Son transfert, pour la durée de l'agon, pourrait affirmer la complémentarité des deux

\footnotetext{
81 Voir M. Detienne, dans M. Detienne et G. Sissa, La vie quotidienne des dieux grecs, Paris, 1989, p. 246.

${ }^{82}$ L'identification archéologique du sanctuaire, longtemps discutée, est désormais assurée, à Dhilesi, sur le littoral. Voir Ch. I. PITÉRos, «Excavations of a Stoa at Delesi and the Site of the

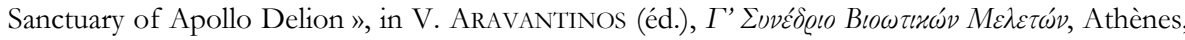
2000, p. 357-359. Les Tanagréens semblent avoir eu le contrôle du sanctuaire pendant une grande partie de leur histoire (Pausanias, IX, 20, 1, et X, 28, 6; Strabon, IX, 2, 7) à l'exception de quelques intermittences thébaines (Hérodote, VI, 118). Voir A. SCHACHTER, Cults of Boiotia. 1. Acheloos to Hera, London, 1981 (BICS, Suppl. 38.1), p. 46-47. Sur l'étroitesse des relations entre les deux rives de l'Euripe, voir U. von Wilamowitz, Hermes 21 (1886), p. 91-115, J.K. DavieS, Athenian Propertied Families, Oxford, 1971, p. 472-73 et L. Breglia PulCI, « Demeter tra Eubeo e Beozia », in Recherches sur les cultes grecs et l'occident 2, Naples, 1984, p. 69-88.

${ }^{83} \mathrm{Il}$ s'agit des comptes des agonothètes, dont Cédric Brélaz prépare la publication pour le BCH 2007. Nous remercions le Prof. Ducrey qui nous a permis de prendre connaissance de ce document.

${ }^{84}$ Sans doute par le koinon avant sa dissolution.

${ }^{85}$ L. 23-24.

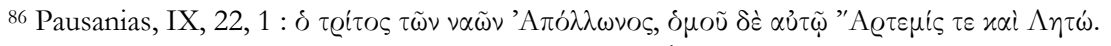

87 J.-L. Durand, «Images pour un autel », in R. Étienne, M.-Th. Le Dinahet (éds), L'espace sacrificiel, Lyon/Paris, 1991, p. 49.
} 
sanctuaires et articuler les relations entre centre et marges du territoire tanagréen sur un mode très différent de ce que nous avons repéré du côté d'Hermès, en marquant la relative dépendance rituelle du sanctuaire littoral vis-à-vis de son homologue urbain et en soulignant la présence à Délion d'un Apollon quasi "poliade $»^{88}$. Les mythes de fondation du Délion s'inscrivent dans le corpus plus large des récits, nettement plus panhelléniques, relatifs aux cheminements d'Apollon de Délos à Delphes ${ }^{89}$. Mais le trait peut-être le plus significatif de l'Apollon «délien » de Tanagra, en son temple urbain, est son association avec Thémis et Aphrodite en un panthéon éminemment politique ${ }^{90}$ alors qu'Hermès semble plutôt en charge des passages et des transitions vers la cité.

Le mythe d'Hermès Promachos définit un espace proprement éphébique, aux marges du territoire mais aussi, symboliquement, aux marges d'un statut de pleine citoyenneté, tout en créant, par un jeu d'anticipation et de retournement, les conditions d'appartenance des jeunes gens à la communauté tanagréenne. Si les éphèbes opérant dans le champ de la ruse avec leur strigile devenu arme se situent aux antipodes du modèle du combat hoplitique, leur élan agonistique et leur victoire, magnifiés par l'épiphanie du dieu, les qualifient pour accomplir le passage auquel Hermès les convie, vers leur pleine intégration dans la polis. Les vertus apprises au gymnase et exercées dans les confins les destinent à prendre place dans la cité d'où, depuis son sanctuaire, le dieu éphèbe a veillé sur leur éducation. Si l'on considère les divers aspects du dieu pris en charge par le sanctuaire, Hermès Promachos apparait comme une puissance complexe qui conjoint l'arbousier ambigu de l'enfance à la figure de l'éphèbe ${ }^{91}$. Engagé dans la cohésion politique et territoriale de Tanagra, susceptible de patronner les étapes de la formation civique, il introduit un lien mobile entre les diverses composantes de la cité, qu'il s'agisse des rapports entre classes d'âge (enfance, adolescence) ou de l'articulation des différents types d'espace qui constituent le territoire. Centre urbain et zones périphériques - arrière-pays montagnard, littoral maritime, terrains de parcours - sont les points névralgiques de la géo-

${ }^{88}$ Les sources disponibles ne permettent pas de définir quelles divinités étaient tenues pour poliades.

89 Pindare, fr. 286, qui fait passer Apollon par Tanagra; Hérodote, VI, 118. Voir SCHACHTER, o.c. (n. 82), p. 45, et pour une lecture des cheminements d'Apollon, M. Detienne, Apollon le couteau à la main, Paris, Gallimard, 1999, notamment p. 19-39.

${ }^{90}$ Pausanias, IX, 22, 1 : le périégète associe étroitement les trois temples voisins qui semblent avoir occupé une position éminente, sinon prééminente dans la partie haute de la cité. Voir M. Detienne, «Les dieux du politique », Annuaire EPHE Ve section 103 (1994-95), p. 237-239.

91 L'iconographie des monnaies tanagréennes d'époque impériale semble synthétiser les différents aspects du mythe du Promachos (voir F. ImHOOF-Blumer et P. GARdNer, Ancient Coins Illustrating Lost Masterpieces of Greek. Art. A Numismatic Commentary on Pausanias, Chicago, 1964, type $1: \mathrm{X}, \mathrm{XIV} ; 2$ : X, XV, XVI). Le dieu éphèbe (nu, imberbe, cheveux courts) est associé à l'andrachnos qui épouse le galbe de son corps, le mouvement du regard et le geste du caducée, tenu à l'horizontale, semble indiquer un passage, auquel l'aigle posé sur l'arbuste pourrait donner sens, s’il renvoie bien à Zeus. 
graphie de la cité entre lesquels se déploie l'organisation complexe de la chora, et qu'il importe de tenir ensemble en un cosmos uni, à l'égal du corps politique. Le sanctuaire d'Hermès Promachos semble assumer de manière privilégiée les relations de la polis avec ses différents eschatiai, l'oros qui porte la mémoire de l'âge d'or et que parcourent les bergers, et le bord de mer où se joue la défense de Tanagra contre les Eubéens et où s'éprouvent les vertus guerrières des éphèbes.

Aucun schéma simple, aisément généralisable, ne suffit à rendre compte d'une organisation de l'espace dont les structures panthéoniques révèlent la richesse. Dans la petite cité béotienne, certains aspects de la mise en œuvre spatiale des transitions éphébiques sont prises en charge par une autre figure d'Hermès, le Criophoros. Le mythe résumé par Pausanias ${ }^{92}$ se présente comme l'aition d'un rite qui entraîne les éphèbes aux limites de la cité. Mais, cette fois, sont en jeu non les confins du territoire mais les remparts de la ville. Le dieu passe pour avoir détourné (apotrepsal) un loimos en portant un bélier sur ses épaules autour des murs de la cité. En retour, les Tanagréens ont érigé une statue d'Hermès criophore - figuré en éphèbe, imberbe et nu ${ }^{93}$ - et institué un rituel éphébique. Chaque année, lors de la fête des Hermaia, «l'éphèbe le plus beau quant à l'allure (to eidos) fait le tour complet des remparts en tenant un agneau sur ses épaules ». Le récit suggère la figure d'un Hermès purificateur qui réactive, au lieu "hermaïque» des échanges et des passages, la puissance protectrice des limites - tracées, fondées, «apolliniennes » - que constitue aussi l'enceinte. Rien toutefois n'indique que le dieu sépare le pur et l'impur ${ }^{94}$, qu'il délimite ou réserve un espace, ainsi que le suggérerait une interprétation trop univoque - et trop «apollinienne »- des rites de circumambulation qui ont, d'ordinaire, pour fonction de purifier, séparer ou consacrer l'espace autour duquel ils sont accomplis95. Au contraire, l'épiphanie d'Hermès autour des remparts produit des effets qui s'étendent à l'ensemble du territoire tanagréen. Il faut prendre le texte de Pausanias à la lettre, qui, dans sa concision mythographique, schématise le mode d'action du dieu : Hermès écarte le fléau par son seul passage, il le détourne sans pour autant instaurer une limite infrangible.

Un point reste à éclaircir. Quel rapport faut-il établir entre la dimension éphébique du rituel et l'apparition d'un Hermès berger et purificateur autour

92 Pausanias, IX, 22, 1. Cf. supra.

${ }^{3}$ La statue, œuvre de Calamis, est connue par des monnaies tanagréennes d'époque impériale, ImHoOf-Blumer, o.c. (n. 91), 115 n 5, pl. X 11 et 12; J. Frel, GettyMusJ 1 (1974), p. 55-57. L'Hermès Barracco, Rome, Mus. Barr. 83, copie romaine d'un original archaïsant du Irer s. av. J.C., reste la figure la plus évocatrice du criophoros tanagréen (le scepticisme de G. SIEBERT, LIMC, Hermès 285, nous semble excessif).

94 R. PARker, Miasma, Oxford, 1983, chap. I, « Purification: a Science of Division », en particulier p. 21-22.

95 J. RUDHARDT, Notions fondamentales de la pensée religieuse et actes constitutifs du culte, Paris, $1992^{2}$ [1958], p. 167, 170, 228-229; L. GERNET, Droit et institutions en Grèce antique, Paris, $1982^{2}$ [1968], p. $70-75$. 
des remparts ? Il nous semble suggéré dans l'écart, souligné par Pausanias, entre l'action mythique du dieu et la pratique rituelle. Lors de la cérémonie, le plus bel éphèbe porte un agneau alors que le dieu, lors de son épiphanie, portait un bélier. Dans l'imaginaire animalier grec, le bélier est d'abord le chef qui conduit le troupeau ${ }^{96}$. Artémidore, dans sa Clef des songes, fait même dériver krios de kreien, commander ${ }^{97}$. Désigné comme «le plus beau ", l'éphèbe qui accomplit le rite a été choisi lors d'une kallisteria, un concours de beauté, destiné à sélectionner celui que ses vertus (euandria, euaxia, enoplia, eutaxia...) prédisposent le mieux à prendre pleinement part à la cité, pour la défendre, voire, un jour, la conduire. L'éphèbe est encore dans une position analogue à l'agneau qu'il porte, tandis qu'Hermès, l'episkopos gymnasion, est, par excellence, le guide qui mène la jeunesse vers la cité. C'est à sa limite même que s'accomplit le rite, avant que les éphèbes n'y acquièrent leur statut définitif d'hommes et de citoyens. C'est dire que l'agneau, pas plus que le bélier du dieu, ne saurait porter les souillures de la cité, il est au contraire lié à son avenir, à la promesse de son renouvellement. Loin d'être expulsé comme un pharmakon ${ }^{98}$, il devait plutôt être sacrifié au Criophoros, dans son sanctuaire, au cœur de la cité. Au moment des Hermaia, ultime étape avant l'intégration d'une génération nouvelle, les murs opèrent à la fois comme la limite entre deux espaces et entre deux statuts, et comme le point de transition hermaïque, le lieu d'un passage préparé entre eschatia et gymnase. Le couronnement d'une éphébie réussie qu'est la circumambulation autour des remparts a été anticipé par une tournée des sanctuaires balisant le territoire, à laquelle les jeunes Tanagréens sont astreints durant leur éphébie, à l'époque de Pausanias du moins ${ }^{99}$.

Dans les mythes de Tanagra, les remparts sont régulièrement mis en relation avec la menace d'une souillure susceptible d'anéantir, de stériliser la cité. C'est pourquoi la marche giratoire du " plus bel éphèbe » et l'aition auquel elle répond, doivent être replacés dans un complexe plus large mettant en jeu l'ensemble des relations qui se nouent entre murs de la ville, souillure, pratiques de circumambulation et avenir de la cité. Dans un des récits de fondation de Tanagra, Poimandros, le bouvier éponyme d'un des noms "poétiques» de Tanagra, «Poimandra, la cité aux belles vaches », accomplit le synœcisme des villages de Tanagride en creusant le fossé qui délimite l'espace de la future cité et en la dotant de murs (eteichise) ${ }^{100}$. Un litige avec l'architecte Polykritos, qui n'a que

\footnotetext{
${ }^{96}$ Detienne, Apollon, cit. (n. 89), p. 88.

${ }^{97}$ Artémidore, Onirocritique II, 12 (119).

${ }^{98}$ Hypothèse reprise entre autres par M.P. NiLSsON, Griechische Feste, Leipzig, 1906, p. 392, et justement réfutée par W. F. Отто, Dionysos. Le mythe et le culte, tr. fr., Paris, 1969, p. 46-47.

${ }^{99}$ IG XII Suppl., 646, revu par L. ROBERT, RPhil 1939, p. 122-128 et OMS II, p. 1275-1281, qui suppose que les Tanagréens ont adopté le modèle attique. Si tel est le cas, ils l'auront intégré sans peine à leur propre pratique.

100 Plutarque, Moralia, 299c-e.
} 
mépris pour le modeste fossé, s'achève par la mort du fil de Poimandros qu'atteint malencontreusement la pierre que le fondateur, dans sa colère, destinait à Polykritos ${ }^{101}$. La mort de l'enfant suscite une souillure : Poimandros, le traceur de limites, doit s'exiler de la cité qu'il a fondée et se faire le suppliant de son ennemi Achille qui assiège la place. Achille l'envoie à Calchis où il sera

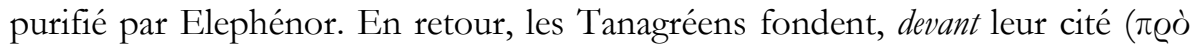
$\tau \tilde{\eta} \varsigma \pi \hat{\lambda} \lambda \varepsilon \varsigma$ ), un ensemble de sanctuaires en l'honneur d'Achille et des autres Achéens qui ont contribué à écarter d'eux la souillure ${ }^{102}$. Mais en rassemblant les Tanagréens en une polis ceinte de remparts, Poimandros leur a assuré une maîtrise durable de leur territoire, au prix de son abandon momentané. Le contrôle de la chora, comme celui du loimos, se joue depuis les murs de la ville. L'épiphanie d'Hermès se produit en un lieu qui est à la fois celui de la fondation de la cité et de la première impureté susceptible d'en menacer l'existence et l'avenir ${ }^{103}$. Éphèbe en charge des éphèbes, puissance de faire croître et décroître troupeaux et cités, il agit sur la fondation en auxidemos ${ }^{104}$. Il fait croître le peuple en écartant le loimos depuis la limite d'où il pourrait se déployer, et en conduisant et guidant les éphèbes qui sont la relève de la cité. En faisant le choix des remparts, Hermès Criophoros renforce, selon son mode propre, la puissance protectrice des limites instituées, il l'étend jusqu'aux confins.

Une autre image des eschatiai tanagréennes se dessine autour de Dionysos, étroitement liée à certains de ses modes et de ses domaines d'action. Dans une des versions de la mise à mort du triton ${ }^{105}$, les confins maritimes sont traités comme un terrain de parcours, le triton ravit le bétail qui pait au bord de la mer et bouscule les petites embarcations. Les Tanagréens lui apporte un cratère de vin qui l'enivre et l'endort, avant de le décapiter. Dans l'autre version ${ }^{106}$, Pausanias raconte - une fois encore - une épiphanie divine qui met en jeu les relations du dieu avec les desservantes de son culte et avec l'élément liquide - la mer, en l'occurrence - d'où il se plait à venir et où il aime se réfugier ${ }^{107}$. Dionysos vient au secours des femmes de Tanagra au moment où elles se purifient dans la mer avant la célébration de ses orgia. Il a répondu à leurs cris et combattu le monstre qui les attaquait. Une tradition relative au héros Eunostos confirme que, pour les Tanagréens, les eschatiai maritimes constituent un espace

101 Variante dans un fragment mutilé d'Aristophane de Béotie (POxy., 2463) où c'est un des fils de Poimandros, Ephippos, qui franchit le fossé.

102 Au temps de Plutarque, les Tanagréens ne gardent la mémoire du nom que du seul sanctuaire d'Achille.

103 Sur les effets du loimos, voir J.L. DuRAND, "Formules attiques du fonder», in M. DETIENNE (éd.), Tracés de fondation, Paris/Louvain, 1990 (Bibliothèque de l'EPHE, Sciences religieuses), p. 271-287.

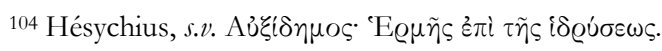

105 Pausanias, IX, 20, 4.

106 Ibid.

107 Voir OTTO, o.c. (n. 98), p. 170-179. 
propice à la purification : un certain Kleidamos, « un homme en vue », avait un jour croisé le héros alors qu'il allait se baigner dans la mer après que son sanctuaire eut été souillé108. Plusieurs représentations des eschatiai maritimes interfèrent et se croisent en des configurations mythiques et panthéoniques d'autant plus riches que la variante tanagréenne du motif «des femmes menacées par un péril venu de la mer » s'insère dans un ensemble régional plus large mettant en jeu les sanctuaires de bord de mer d'Artémis, notamment Brauron ou Halai où la déesse et Dionysos se partagent les fêtes nocturnes ${ }^{109}$.

L'action du Dionysos tanagréen sur les limites pourrait également s'avérer plus complexe. Il semble qu'il faille identifier les orgia du dieu auxquels fait référence Pausanias avec les Nyctélies mentionnées par Plutarque dans son récit de la fondation de Tanagra par Poimandros ${ }^{110}$. La pierre avec laquelle le fondateur vise Polykritos, coupable d'avoir enfreint les limites de la nouvelle cité, et qui fera de lui le meurtrier exilé de son fils « avait été caché là depuis des temps très anciens ('̊x $\pi \alpha \lambda \alpha i o v)$ et placée là en vue des Nyctélies », une fête que Plutarque, bon connaisseur des usages béotiens, associe aux Agrionies dionysiaques ${ }^{111}$. Si tel est bien le cas, le dieu se trouverait intervenir, à l'instar d'Hermès, mais en fonction de ses champs de compétences propres (en relation avec les femmes et en expulsant le fondateur, comme à Thèbes), sur les deux limites complémentaires de la cité : les murs de la ville et les confins du territoire.

Tant dans les processus «d'appropriation symbolique » et de balisage du territoire que de définition qualitative des espaces, le mythe fonctionne dans un rapport de complémentarité différentielle avec la pratique rituelle. Il intègre cette dernière à l'énonciation de l'aition, souligne l'écart signifiant entre l'épiphanie divine et le rituel éphébique, écart qui explicite une des fonctions que le dieu assume à l'occasion des Hermaia. Il met en évidence la présence de puissances qui restent étrangères à la pratique rituelle mais n'en déterminent pas moins, ne serait-ce qu'à titre de préalable, des qualités et des propriétés de l'espace nécessaires à la mise en place de la cité : persistance discrète de l'âge d'or dans la chora, position d'un centre cosmique distinct des centres politiques et cultuels... Les schémas récurrents des aitia de Pausanias (l'épiphanie d'un dieu repoussant

\footnotetext{
108 Plutarque, Moralia, 301a, citant Dioclès, Sur les temples des héros, qui rapporte un décret tanagréen.

109 P. Brulé, La fille d'Athènes, Paris, 1987 (Annales littéraires de l'université de Besançon, 363), p. 187-200 et 310-312.

110 Plutarque, Moralia, 299c-e. Voir SCHACHTER, o.c. (n. 82), p. 185 et Roller, Sources, cit. (n. 13), p. 153.

111 Plutarque, de Iside et Osiride, 291a et 364f, où il associe les Nyctélies à des récits relatifs aux Titans. Les interprètes modernes sont peut-être trop prompts à n'y voir, comme le suggère le contexte de relecture osirienne, qu'une allusion au mythe du démembrement de Dionysos. Une monnaie d'époque romaine montre que le temple de Dionysos à Tanagra était décoré d'atlantes, un fait qui serait banal si Atlas et les généalogies atlantides ne jouaient un rôle si important dans la région. Autre arrière-plan titanique à prendre en compte...
} 
ou écartant une menace), où l'on pourrait d'abord soupçonner un effet d'homogénéisation de l'écriture périégiétique, révèlent, dans leurs traits singuliers, une structuration spécifique de l'espace, authentiquement tanagréenne, qui s'est développée comme une pensée particulièrement subtile des articulations entre remparts et confins. On y pressent que les interventions si caractéristiques d'Hermès et de Dionysos - les seules à avoir retenu l'attention de Pausanias s'insèrent dans un complexe plus large de prise en charge du territoire par les puissances du panthéon (Apollon, Déméter ${ }^{112} \ldots$.). Les aitia eux-mêmes se répondent, de l'Hermès Lenkos ${ }^{113}$ dont le culte (oublié au temps de Pausanias ?) est censé avoir été institué après un sacrifice humain mythique assurant l'ancrage durable de la communauté tanagréenne sur son territoire, à l'Hermès Promachos préparant les éphèbes, avenir de la cité, à en assurer la défense.

Au sein de la pratique polythéiste dans laquelle l'un et l'autre prennent sens, l'opposition entre mythe et rite s'estompe. Sans doute dira-t-on plus volontiers que le mythe opère dans le champ de la nomination, de la désignation, du récit ou du schème qui le porte. Mais, pour la compréhension du modus operandi, il importe d'abord de saisir comment élaboration mythique et rite se répondent; comment ils mettent en place, conjointement et différentiellement, les interactions entre panthéon et espaces qualitatifs. De ce point de vue, il est tout aussi pertinent d'appeler mythe le processus (pratique et représentation) qui engendre tant les récits et les rituels ${ }^{114}$ que les paroles et récits qui prolongent, explicitent et interprètent les rites ou leur font contrepoint et se développent en explorations autonomes. En revanche, pour analyser ce qui fonde l'articulation d'une communauté - les Tanagréens et leurs dieux - et d'une terre, il est décisif d'évaluer la place respective du sacrifice, des diverses pratiques rituelles (procession, "offrandes »...), et des pratiques discursives - sachant que ces dernières sont aussi partie prenante des premières ${ }^{115} \ldots$

Dominique JAILLARD

Université de Lausanne

IASA

Anthropoloe 4015

CH - 1015 Lausanne - Dorigny

Courriel:dominique.jaillard@unil.ch

112 Voir le débat, au III ${ }^{\mathrm{e}}$ s. av. J.-C., sur le lieu de reconstruction du sanctuaire de Déméter, S. REINACH, REG 12 (1899), p. 53-115 et A. SCHACHTER, "Reflections on an inscription from Tanagra », in J. BINTLIFF (éd.), Recent Developments in the History and Archaeology of Central Greece, Oxford, 1997, p. 277-286.

113 Scholie à Lycophron, 679.

114 SCHEID - SVENBRO, o.c. (n. 3), p. 11.

115 Voir, par exemple, côté athénien, Durand, l.c. (n. 103) et DeTIENNE, Vie quotidienne, cit. (n. 81), p. 231-252. 